\title{
Assessment of non-standard HIV antiretroviral therapy regimens at Lighthouse Trust in Lilongwe, Malawi \\ BS Barnett ${ }^{* 1,2}$, T Chaweza*3, H Tweya ${ }^{3}$, W Ngambi ${ }^{3}$, S Phiri' ${ }^{3}$, MC Hosseinipour ${ }^{1}$
}

1.UNC Project-Malawi, Lilongwe, Malawi

2.Vanderbilt University School of Medicine, Nashville, Tennessee, USA

3.Lighthouse Trust, Lilongwe, Malawi

*Both authors contributed equally to this work

Correspondence to: Brian S. Barnett :E-mail: bbarnett@partners.org

Abstract

\begin{abstract}
Aim
Lighthouse Trust in Lilongwe, Malawi serves approximately 25,000 patients with HIV antiretroviral therapy (ART) regimens standardized according to national treatment guidelines. However, as a referral centre for complex cases, Lighthouse Trust occasionally treats patients with non-standard ART regimens (NS-ART) that deviate from the treatment guidelines. We evaluated factors contributing to the use of NS-ART and whether patients could transition to standard regimens.
\end{abstract}

\section{Methods}

This was a cross-sectional study of all adult patients at Lighthouse Trust being treated with NS-ART as of February 2012. Patients were identified using the electronic data system. Medical charts were reviewed and descriptive statistics were obtained.

\section{Results}

One hundred six patients were initially found being treated with NS-ART, and 92 adult patients were confirmed to be on NS-ART after review. Mean patient age was $42.4 \pm 10.3$ years, and $52(57 \%)$ were female. Mean duration of treatment with the NS-ART being used at the time of data collection was $2.1 \pm 1.5$ years. Eight patients $(9 \%)$ were on modified first-line NS-ART and $84(91 \%)$ were on modified second-line NS-ART, with 90 patients $(98 \%)$ having multiple factors contributing to NS-ART use. Severe toxicity from one medication contributed in 28 cases $(30 \%)$ and toxicity from multiple medications contributed in 46 cases $(50 \%)$, while 22 patients $(24 \%)$ were transitioned to NS-ART following a stockout of their original medication. Following clinical review, 84 patients $(91 \%)$ were transitioned to standard regimens, and eight (9\%) were maintained on NS-ART because of incompatibility of their clinical features with the latest national guidelines.

\section{Conclusions}

Primary factors contributing to NS-ART use were medication toxicities and medication stockouts. Most patients were transitioned to standard regimens, although the need for NS-ART remains.

\section{Introduction}

Free antiretroviral therapy (ART) for individuals living with HIV was introduced in Malawi in 2004, with nearly 400,000 patients being treated as of September 2012. ${ }^{1}$ ART guidelines have been standardized since programme inception, with common first- and second-line regimens across the country. Despite these guidelines, a small number of patients throughout the country are treated with non-standard ART regimens (NS-ART).

Lighthouse Trust, in Malawi's capital of Lilongwe, serves approximately 25,000 patients living with HIV by providing the aforementioned standardized ART regimens at two clinics. As a referral centre for complex cases, Lighthouse Trust occasionally treats patients with NS-ART. The use of such regimens is largely managed on a case-by-case basis, and there is a lack of synthesized data related to the underlying reasons for using NS-ART. Challenges in quantifying these regimens have resulted in medication stockouts in the past, necessitating further modifications.

The aim of this study was to evaluate the use of NS-ART at Lighthouse Trust in order to determine the reasons for the use of NS-ART and assess whether patients could safely transition to standard ART regimens following the 2011 national HIV treatment guideline change in Malawi.

\section{Methods}

\section{Study design and population}

We conducted a cross-sectional study of routinely gathered data at Lighthouse Trust's two public ART clinics in Lilongwe, Malawi: (1) Lighthouse Clinic and (2) Martin Preuss Centre
(MPC). This study included all patients 18 years of age and older at Lighthouse Trust who were being treated with NSART as of February 2012. ART regimens in the country are standardized according to national treatment guidelines. Prior to 2011, ART regimens in Malawi were dictated by the Guidelines for the Use of Antiretroviral Therapy in Malawi, 3rd Edition. ${ }^{2}$ According to these guidelines, the first-line treatment regimen was with lamivudine, nevirapine, and stavudine, in the form of the fixed-medication combination Triomune. In cases of nevirapine-associated toxicity, efavirenz could be substituted, and in cases of toxicity attributed to stavudine, zidovudine could be substituted. In cases of dual reactions to stavudine and nevirapine, patients were switched to a regimen of zidovudine, lamivudine, and efavirenz. In cases of first-line regimen treatment failure, second-line therapy consisted of zidovudine, lamivudine, tenofovir and lopinavir/ritonavir.

A more recent set of Malawian national guidelines was implemented in July 2011, with first-line ART also consisting of lamivudine, nevirapine, and stavudine in the form of Triomune. ${ }^{3}$ In cases of toxicity, zidovudine or tenofovir could be substituted for stavudine (in cases of severe peripheral neuropathy, pancreatitis, lactic acidosis, or lypodystrophy), and efavirenz for nevirapine (in cases of severe skin reactions or hepatitis). For breastfeeding women, pregnant women, and TB/HIV co-infected patients, efavirenz, lamivudine, and tenofovir became available for first-line therapy. In cases of dual reactions to stavudine and nevirapine, patients were switched to a regimen of efavirenz, lamivudine, and zidovudine. However, individual lamivudine was not available (as it had been prior to 2011). Patients 
failing first-line ART were transitioned to second-line ART, which included lopinavir/ritonavir and either tenofovir or zidovudine combined with lamivudine.

\section{Data collection}

All patients who receive care at Lighthouse and MPC for treatment of HIV are registered in the clinics' electronic data system (EDS). As directed by the national treatment guidelines in place during the study period, all adults who were HIV-seropositive were initiated on ART if they had a CD4-lymphocyte count $\leq 350$ cells $/ \mathrm{mm}^{3}$ or were assessed to be in WHO clinical stages III or IV. Using EDS data, we retrospectively identified 106 patients as being treated with NS-ART as of February 28, 2012 and subsequently performed chart reviews. Data about patient demographics, treatments, and reasons for alterations in treatment were extracted and managed using REDCap electronic data capture tools. ${ }^{4}$

\section{Statistical analysis}

Descriptive statistics were used to describe the characteristics of the study population and were obtained using Stata ${ }^{\circledR}$ version 11.0 (Stata Corporation, College Station, Texas, USA).

\section{Ethical considerations}

The study proposal was approved by the Malawi National Health Sciences Research Committee and the School of Medicine Institutional Review Board at the University of North Carolina at Chapel Hill. Additionally, all records used in this study were de-identified prior to being analysed.

\section{Results}

Of the 17,000 patients being treated at Lighthouse Trust for HIV in February 2012, 106 were identified in the EDS as being treated with NS-ART, and their files were reviewed. Upon further investigation, it was noted that 12 cases were mistakenly identified as using NS-ART, leaving 94 confirmed patients being treated with NS-ART. Two patients were excluded because they were less than 18 years of age, leaving 92 patients eligible for this study (Table 1).

\section{Table 1: Patient demographic and clinical characteristics}

\begin{tabular}{ll}
\hline Patient demographic and clinical characteristics & (N = 92) \\
\hline & mean \pm SD \\
\cline { 2 - 2 } Age (years) & $42.4 \pm 10.3$ \\
CD4 count at initiation (cells $\left./ \mathrm{mm}^{3}\right)$ & $213 \pm 116$ \\
Duration of ART (years) & $5.6 \pm 3.4$ \\
Duration of treatment on any NS-ART (years) & $2.2 \pm 1.5$ \\
Duration of treatment on current NS-ART (years) & $2.1 \pm 1.5$ \\
\hline & $\mathbf{n ~ ( \% )}$ \\
\cline { 2 - 2 } Female gender & $52(57)$ \\
WHO Stage III & $59(64)$ \\
WHO Stage IV & $8(9)$ \\
CD4 count $\leq 350$ cells $/$ mm ${ }^{3}$ & $25(27)$ \\
Being treated with first-line NS-ART & $8(9)$ \\
Being treated with modified second-line NS-ART & $84(91)$ \\
\hline SD = standard deviation; ART $=$ antiretroviral therapy; & \\
NS-ART $=$ non-standard ART regimen &
\end{tabular}

Mean patient age was $42.4 \pm 10.3$ years and 52 patients $(57 \%)$ were female. Fifty-nine patients (64\%) had been initiated on ART after being designated as having WHO clinical stage III disease, while eight $(9 \%)$ had WHO clinical stage IV disease, and $25(27 \%)$ had either WHO clinical stage I or II disease with a CD4-lymphocyte count $\leq 350$ cells $/ \mathrm{mm}^{3}$.

Patients were being treated with twenty different nonstandard combinations of ART, and these regimens were usually the third ART regimens used during the course of their HIV management. The most common NS-ARTs used are listed in Table 2.

Table 2: Most common non-standard antiretroviral therapy regimens

\begin{tabular}{ll}
\hline Regimen & n (\%) \\
\hline lopinavir/ritonavir monotherapy & $30(33)$ \\
stavudine + lamivudine + lopinavir/ritonavir & $21(23)$ \\
zidovudine + lamivudine + tenofovir + lopinavir/ritonavir* & $11(12)$ \\
lopinavir/ ritonavir + tenofovir & $5(5)$ \\
Other & $27(29)$ \\
\hline *Regimen was standard in 2008 guidelines but did not conform to 2011 guidelines
\end{tabular}

The most common contributing factor for being transitioned to NS-ART was severe toxicity from multiple antiretroviral (ARV) medications, which affected 46 patients (50\%). Additional contributory factors are detailed in Table 3.

Table 3: Reasons for patients being managed with non-standard antiretroviral therapy regimens

\begin{tabular}{ll}
\hline Reason & n (\%)* \\
\hline Severe toxicity from multiple antiretroviral medications & $46(50)$ \\
Severe toxicity from one antiretroviral medication & $28(30)$ \\
Medication stockout while on standard regimen & $22(24)$ \\
Transferred in from an antiretroviral therapy study & $22(24)$ \\
Treatment according to previous guidelines & $12(13)$ \\
Pregnancy & $6(7)$ \\
Other & $5(5)$ \\
\hline
\end{tabular}

*Percentages total greater than 100 because most patients were on non-standard regimens for more than of these reasons

The medications most likely to induce toxicities contributing to NS-ART use were stavudine (35 patients, 38\%), nevirapine (29 patients, 32\%), efavirenz (26 patients, $28 \%$ ), zidovudine (21 patients, 23\%), and tenofovir (13 patients, 14\%). Nearly all patients ( $\mathrm{n}=90 ; 98 \%$ ) had multiple contributing factors for eventually being switched to treatment with NS-ART.

Following chart review, 84 patients (91\%) were transitioned to a standard ART regimen according to the 2011 guidelines, and eight $(9 \%)$ were maintained on NS-ART. Of those who were maintained on NS-ART, three (37.5\%) were on a modified second-line NS-ART because of toxicity attributed to lopinavir/ritonavir, while the other five $(62.5 \%)$ demonstrated toxicity associated with all or all but one of the standard nucleoside reverse transcriptase inhibitors (NRTIs), which include lamivudine, stavudine, zidovudine, and tenofovir. Notably, none of the patients in the latter group had ever been treated with abacavir, which is also an NRTI, but was only included in 2011 Malawian treatment guidelines for children and not adults.

\section{Discussion}

Less than $1 \%$ of the 17,000 patients receiving ART for treatment of HIV at Lighthouse Trust in 2012 were being treated with NS-ART, signifying a strong adherence to standardized regimens by clinicians. Assessing the reasons for use of NS-ART is essential to ensuring that optimal patient care is provided and that the clinics' adherence to national guidelines remains high. The use of NS-ART at Lighthouse Trust is multifactorial in origin, with clinic-level factors, limitations of national guidelines for individualized 
regimens, and medication supply ordering challenges being major contributors. Overall, use of NS-ART did not suggest suboptimal care but rather limitations of the current repertoire of ARV medications, which leads to difficulties managing patients with medication toxicities.

The contribution of medication shortages illustrates the impact of uncertainty in Malawi's ART supply chain. Malawi has struggled with national medication stockouts in the past, especially as the number of different ART regimens in use has increased, ${ }^{5}$ despite the expectation that expansion of available regimens would reduce the percentage of patients needing treatment with NS-ART because of medication toxicities. Though stockouts are uncommon for medications that are part of standardized regimens, challenges remain with predicting the necessary quantities of non-standard medications being used, and this uncertainty often leads to further alterations of NS-ARTs. Prior to regular access to tenofovir/lamivudine as a fixed-dose combination within the programme, individual supplies of tenofovir, lamivudine, and the non-nucleoside reverse transcriptase inhibitor (NNRTI) were required for tenofovir to be used when faced with stavudine and zidovudine toxicity. Any breach in the supply chain to the individual components could lead to potentially suboptimal regimens being provided as an interim solution in response to a short-term medication stockout. At the clinic level, one point of intervention would be to regularly review the medication regimens for each patient at every visit to ensure that an NS-ART that may have initiated because of a stockout is no longer continued after the stockout has resolved.

Though most patients had multiple reasons for treatment with NS-ART, most of them could still be transitioned to standard regimens after review. Given the small number of patients (92) in our clinics being treated with NS-ART at the time of this investigation, our study is limited by its small sample size, as well as its retrospective nature, which may have led to the omission of some factors contributing to the use of NS-ART. Still, the need for NS-ART remains in some of the cases reviewed here, suggesting a potential role for additional medications for toxicity management, such as abacavir in adults, or an alternative to lopinavir/ritonavir, or the addition of the integrase inhibitor class of drugs. Notably, the 2014 Malawi guidelines address many of the shortfalls noted in the 2008 and 2011 guidelines: abacavir/lamivudine has been added as an NRTI backbone for both adults and children, and atazanavir/ritonavir has become the protease inhibitor combination of choice for second-line treatment, with lopinavir/ritonavir now serving as an alternative. ${ }^{6}$ This is particularly important since nearly half of patients being treated with NS-ART were on second-line therapy. With only one standard second-line treatment regimen available prior to 2014, any additional medication toxicity resulted in the use of NS-ART, either by discontinuation of the problematic drug or by its replacement.

Table 4 compares the ARV medication formulations available in Malawi in 2008, 2011, and 2014.,3,3 In addition to combination formulations, previously there were also single medication formulations available for use in NSARTs. A remaining shortfall of the current 2014 guidelines exists in the extremely rare situation of lamivudine toxicity, most commonly manifested as red cell aplasia, ${ }^{7}$ or in patients with renal failure, for whom NRTI dose reductions may not be possible with fixed-dose combinations. In second- line therapy, the use of raltegravir with lopinavir/ritonavir, an NRTI-sparing regimen, is effective and may provide treatment options for those unable to tolerate NRTIs. ${ }^{8,9}$ The maintenance of ART for those requiring individualized treatment poses a challenge for the HIV programme. This potential stumbling block likely can be managed by the use of specialized ordering through centres of excellence given the relatively small scope at present.

In summary, our analysis underscores the need to critically evaluate existing patients' ART regimens as guidelines change to ensure they have access to sustainable, effective ART and to support the use of Malawi's standardized ART treatment guidelines.

Table 4: A comparison of antiretroviral (ARV) medication formulations available in Malawi in years when national guidelines were updated

\begin{tabular}{|c|c|c|c|}
\hline ARV medication formulation & 2008 & 2011 & 2014 \\
\hline abacavir & $\mathrm{P}$ & & \\
\hline abacavir/lamivudine & & $\mathrm{P}$ & $\mathrm{X}$ \\
\hline atazanavir/ritonavir & & & $\mathrm{X}^{*}$ \\
\hline didanosine & $\mathrm{P}$ & & \\
\hline efavirenz & $\mathrm{X}$ & $\mathrm{X}$ & $\mathrm{X}$ \\
\hline efavirenz/lamivudine/tenofovir & & $\mathrm{X}$ & $\mathrm{X}$ \\
\hline lamivudine & $\mathrm{X}$ & & \\
\hline lamivudine/nevirapine/stavudine & $\mathrm{X}$ & $\mathrm{X}$ & $\mathrm{X}$ \\
\hline lamivudine/nevirapine/zidovudine & & $\mathrm{X}$ & $\mathrm{X}$ \\
\hline lamivudine/stavudine & $\mathrm{X}$ & $\mathrm{X}$ & $\mathrm{X}$ \\
\hline lamivudine/tenofovir & & $\mathrm{X}$ & $\mathrm{X}$ \\
\hline lamivudine/zidovudine & $\mathrm{X}$ & $\mathrm{X}$ & $\mathrm{X}$ \\
\hline lopinavir/ritonavir & $\mathrm{X}$ & $\mathrm{X}$ & $\mathrm{P} * *$ \\
\hline nevirapine & $\mathrm{X}$ & $\mathrm{X}$ & $\mathrm{X}$ \\
\hline tenofovir & $\mathrm{X}$ & & \\
\hline
\end{tabular}

*The atazanavir/ritonavir formulation became available in Malawi in April 2013, prior to the release of the 2014 guidelines.

**Lopinavir/ritonavir is also available for treatment of adults with HIV and tuberculosis during simultaneous antiretroviral and rifampicin-based antituberculosis treatment.

\section{Case 1}

A 60-year-old male, who was diagnosed with HIV in June 2005 and had a baseline CD4 count of 138 cells $/ \mathrm{mm}^{3}$, serves as the first example. He initiated ART with a fixeddose combination of lamivudine, nevirapine, and stavudine. He developed hepatitis secondary to nevirapine, which was replaced with efavirenz in January 2010, while maintaining the stavudine and lamivudine. A few months later, in May 2010, he developed depression, confusion, and insomnia. This was suspected to be caused by efavirenz, which was replaced with lopinavir/ritonavir. Unfortunately, he was not able to tolerate this change because of the resulting gastrointestinal upset. Around this time, he also developed peripheral neuropathy believed to be secondary to stavudine. Finally, in September 2010, his regimen was changed to lamivudine, tenofovir, and zidovudine (a three-NRTI combination) because of the lack of alternatives to lopinavir/ritonavir. For ART-naïve patients, three-NRTI regimens can maintain HIV-RNA suppression, ${ }^{10,11}$ although not as efficaciously as regimens containing two NRTIs and an NNRTI. After our study this MMJ VOL 28 (1): March 2016 
patient was initially maintained on this regimen. He could only be transitioned to a standard regimen when atazanavir/ ritonavir became available to the ART programme in April 2013. He thereafter was managed on atazanavir/ritonavir, lamivudine, and tenofovir.

\section{Case 2.}

The second example is a female patient who was 50 years old during the study period. She was diagnosed with HIV (WHO stage III) in February 2005. ART was initiated with lamivudine, nevirapine, and stavudine. In October 2006 stavudine was replaced with zidovudine after she experienced peripheral neuropathy. However, in September 2008 the patient's zidovudine was replaced with abacavir because she developed anaemia. Thereafter, the patient's abacavir was discontinued after she experienced severe GI intolerance (presumed to be hypersensitivity) and her regimen was changed to lopinavir/ritonavir, lamivudine, and tenofovir. However, in February 2010 the patient developed renal failure secondary to tenofovir and was placed on lopinavir/ ritonavir monotherapy. She was maintained on this after review. Monotherapy regimens have been shown to effect reasonable virologic suppression and clinical outcomes, although a combination of a protease inhibitor and integrase inhibitor would be preferred in such an individual with multiple NRTI toxicities ${ }^{8,12,13}$.

\section{Case 3.}

The final example is a female patient who was 44 years old during data collection. She was diagnosed with HIV in March 2009. Her initial CD4 count was 236 cells/ mm3. ART was initiated with lamivudine, nevirapine, and stavudine. She experienced a rash, attributed to nevirapine, after which nevirapine was replaced with efavirenz in May 2009. However, in the following months she developed confusion, which was attributed to efavirenz. In August 2009 efavirenz was discontinued and replaced with lopinavir/ ritonavir. After review, the patient's non-standard regimen (lamivudine, lopinavir/ritonavir, and stavudine) was changed to a standardized regimen of lopinavir/ritonavir, lamivudine, and tenofovir.

\section{Acknowledgements}

The study was supported by funding from the University of North Carolina Center for AIDS Research (P30 AI50410) and the National Institutes of Health (NIH) Office of the Director; Fogarty International Center; Office of AIDS Research; National Cancer Institute; National Eye Institute; National Heart, Lung, and Blood Institute; National Institute of Dental \& Craniofacial Research; National Institute on Drug Abuse; National Institute of Mental Health; National Institute of Allergy and Infectious Diseases; and NIH Office of Research on Women's Health through the Fogarty International Clinical Research Scholars and Fellows Program at Vanderbilt University (R24 TW007988) and the American Relief and Recovery Act. BSB was the recipient of a Fulbright-Fogarty Fellowship in Public Health (2011-12).

We thank the administration, patients and staff of the Lighthouse Clinic and Martin Preuss Centre, who enabled us to carry out this investigation.

\section{References}

1. Ministry of Health, Malawi.Integrated HIV program report JulySeptember 2012. Lilongwe: Ministry of Health, Malawi; 2012.

2. Ministry of Health, Malawi. Guidelines for the use of antiretroviral therapy in Malawi. 3rd ed. Lilongwe: Ministry of Health, Malawi; 2008.

3. Ministry of Health, Malawi. Clinical management of HIV in children and adults. 1st ed. Lilongwe: Ministry of Health, Malawi; 2011.

4. Harris PA, Taylor R, Thielke R, Payne J, Gonzalez N, Conde JG. Research electronic data capture (REDCap)--a metadata-driven methodology and workflow process for providing translational research informatics support. J Biomed Inform. 2009 Apr;42(2):377-81. doi: 10.1016/j.jbi.2008.08.010.

5. Schouten EJ, Jahn A, Ben-Smith A, Makombe SD, Harries AD, Aboagye-Nyame F, et al. Antiretroviral drug supply challenges in the era of scaling up ART in Malawi. J Int AIDS Soc. 2011 Jul 6;14 Suppl 1:S4. doi: 10.1186/1758-2652-14-S1-S4.

6. Ministry of Health, Malawi. Clinical management of HIV in children and adults. 2nd ed. Lilongwe: Ministry of Health, Malawi; 2014.

7. Nakamura K, Tateyama M, Tasato D, Haranaga S, Tamayose M, Yara $\mathrm{S}$, et al. Pure red cell aplasia induced by lamivudine without the influence of zidovudine in a patient infected with human immunodeficiency virus. Intern Med. 2014;53(15):1705-8

8. Paton NI, Kityo C, Hoppe A, Reid A, Kambugu A, Lugemwa A, et al. Assessment of second-line antiretroviral regimens for HIV therapy in Africa. N Engl J Med. 2014 Jul 17;371(3):234-47. doi: 10.1056/ NEJMoa1311274.

9. SECOND-LINE Study Group, Boyd MA, Kumarasamy N, Moore CL, Nwizu C, Losso MH, Mohapi L, et al. Ritonavir-boosted lopinavir plus nucleoside or nucleotide reverse transcriptase inhibitors versus ritonavir-boosted lopinavir plus raltegravir for treatment of $\mathrm{HIV}-1$ infection in adults with virological failure of a standard first-line ART regimen (SECOND-LINE): a randomised, open-label, non-inferiority study. Lancet. 2013 Jun 15;381(9883):2091-9. doi: 10.1016/S01406736(13)61164-2.

10. DART Trial Team, Mugyenyi P, Walker AS, Hakim J, Munderi P, Gibb DM, Kityo C, et al. Routine versus clinically driven laboratory monitoring of HIV antiretroviral therapy in Africa (DART): a randomised non-inferiority trial. Lancet. 2010 Jan 9;375(9709):123-31. doi: 10.1016/S0140-6736(09)62067-5.

11. Gulick RM, Ribaudo HJ, Shikuma CM, Lustgarten S, Squires KE, Meyer WA 3rd, et al. Triple-nucleoside regimens versus efavirenzcontaining regimens for the initial treatment of HIV-1 infection. N Engl J Med. 2004 Apr 29;350(18):1850-61.

12. Bartlett JA, Ribaudo HJ, Wallis CL, Aga E, Katzenstein DA, Stevens WS, et al. Lopinavir/ritonavir monotherapy after virologic failure of first-line antiretroviral therapy in resource-limited settings. AIDS. 2012 Jul 17;26(11):1345-54. doi: 10.1097/QAD.0b013e328353b066.

13. Kumarasamy N, Aga E, Ribaudo H2, Wallis CL, Katzenstein DA, Stevens WS, et al. Lopinavir/ritonavir monotherapy as second-line antiretroviral treatment in resource-limited settings: Week 104 analysis of AIDS Clinical Trials Group (ACTG) A5230. Clin Infect Dis. 2015 May 15;60(10):1552-8. doi: 10.1093/cid/civ109. 